\title{
Interleukin-1 $\beta$ increases permeability and upregulates the expression of vascular endothelial-cadherin in human renal glomerular endothelial cells
}

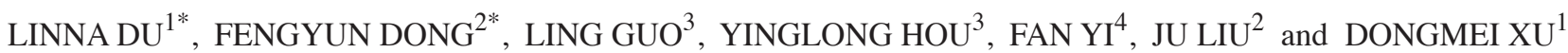 \\ ${ }^{1}$ Department of Nephrology; ${ }^{2}$ Laboratory of Microvascular Medicine, Medical Research Center; \\ ${ }^{3}$ Department of Cardiology, Shandong Provincial Qianfoshan Hospital, Shandong University, Jinan, \\ Shandong 250014; ${ }^{4}$ Department of Pharmacology, Shandong University \\ School of Medicine, Jinan, Shandong 250012, P.R. China
}

Received March 24, 2014; Accepted November 20, 2014

DOI: $10.3892 / \mathrm{mmr} .2015 .3172$

\begin{abstract}
The renal glomerular capillary endothelium is part of the glomerular filtration barrier and is involved in acute and chronic inflammation of the glomerulus. Glomerular endothelial cells are a unique type of microvascular cell, which remain to be fully characterized. The aim of the present study was to examine the permeability of glomerular endothelial cells and their responses to interleukin (IL)-1 $\beta$, a pro-inflammatory cytokine. Human glomerular endothelial cell (HRGEC) and human umbilical vein endothelial cell (HUVEC) monolayers were examined using a Transwell permeability assay, transendothelial electrical resistance (TEER) and by determining the expression of the adhesion molecule, vascular endothelial (VE)-cadherin, in the absence or presence of $10 \mathrm{ng} / \mathrm{ml} \mathrm{IL-1 \beta}$. Compared with the HUVECs, the HRGECs demonstrated higher permeability, lower TEER and reduced expression of VE-cadherin. IL-1 $\beta$ induced an increase in the permeability and a decrease in the TEER of the HRGECs, however, to a lesser extent compared with the HUVECs. Following IL-1 $\beta$ treatment, the expression of VE-cadherin was increased in the HRGECs and decreased in the HUVECs. These results
\end{abstract}

Correspondence to: Professor Dongmei Xu, Department of Nephrology, Shandong Provincial Qianfoshan Hospital, Shandong University, 16766 Jingshi Road, Jinan, Shandong 250014, P.R. China E-mail: xudongmei63@163.com

Professor Ju Liu, Laboratory of Microvascular Medicine, Medical Research Center, Shandong Provincial Qianfoshan Hospital, Shandong University, 16766 Jingshi Road, Jinan, Shandong 250014, P.R. China

E-mail: ju.liu@sdu.edu.cn

${ }^{*}$ Contributed equally

Key words: glomerular endothelial cells, interleukin-1 $\beta$, vascular endothelial-cadherin, permeability suggested that HRGECs have distinct biological properties and specific gene expression features in response to IL-1 $\beta$.

\section{Introduction}

The filtration barrier of the renal corpuscle is composed of the fenestrated endothelium of the glomerular capillaries, fused basal lamina of the endothelial cells and podocytes (1). The barrier permits the passage of water, ions and small molecules from the bloodstream into Bowman's space and restricts the passage of large molecule proteins (2). The glomerular endothelium is a primary contributor to the glomerular filtration barrier and is involved in several pathophysiological processes in the glomerulus $(1,3,4)$. Unlike the most common type of capillary, which has a continuous endothelium, the glomerular capillaries form a large fenestrated area constituting between 20 and $50 \%$ of the entire endothelial surface (5). The renal glomerular endothelial cell (GEC) is a unique microvascular cell type (6). GECs are exposed to all the circulating blood elements and constitutively synthesize bioactive molecules (6). The function of GECs is sensitive to various inflammatory and thrombotic agents, including tumor necrosis factor (TNF)- $\alpha$, interleukin (IL) $1-\beta$, IL-10, IL-6, thrombin and transforming growth factor (TGF)- $\beta 1(7,8)$. Injury to the GECs affects mesangial cells and is significantly involved in the progression of glomerular diseases (9). In addition, the loss of GECs results in sclerosis during glomerular repair (10). However, the biological properties and functional mechanisms of GECs remain to be fully elucidated.

IL-1 $\beta$, a pro-inflammatory cytokine, is a member of the IL-1 family and contributes to the pathological processes of several kidney diseases (11). In particular, IL-1 $\beta$ is associated with the development of common chronic kidney disease complications and renal failure $(12,13)$. It is produced primarily by monocytes, macrophages, dendritic cells, B-lymphocytes, natural killer cells and endothelial cells (11). Following synthesis as a cytosolic precursor (31 kD), it is released as an active cytokine $(17 \mathrm{kD})$ following cleavage by neutrophilic enzymes, including caspase-1 and proteinase-3 (14). There are two receptors known to bind with IL-1, which differ in size and 
distribution (15). The type I receptor is primarily responsible for transmitting the inflammatory effects of IL-1, while the type II receptor binds tightly to IL- $1 \beta$ without activating the downstream signals due to the absence of a cytoplasmic domain (15).

In the human body, IL-1 $\beta$ is secreted into the circulation and directly interacts with the blood vessel endothelium (16). IL-1 $\beta$ is involved in vascular inflammation, hemodynamics and angiogenesis (17). The primary effect of IL-1 $\beta$ on the endothelium is to increase vascular permeability, which has been observed in the endothelial cells from various vascular beds (16). The exposure of human brain microvessel endothelial cells to various cytokines, including IL-1 $\beta$, decreases the transendothelial electrical resistance (TEER), predominantly by increasing the permeability of the tight junctions (18). The permeability of human umbilical vascular endothelial cell (HUVEC) monolayers to fluorescent-labeled albumin can be mediated significantly by IL-1 $\beta$ (19). In addition, IL-1 $\beta$ increases the permeability of pulmonary artery endothelial cells via $\alpha v \beta 5$ integrin- and RhoA-dependent mechanisms (20).

Despite the important role of IL-1 $\beta$ in vascular inflammation, its effects on the glomerular endothelial intercellular junction barrier function remain to be elucidated. The aims of the present study were to characterize the barrier function of glomerular endothelial cells and their responses to IL-1 $\beta$. The permeability of human renal glomerular endothelial cells (HRGEC) and HUVECs were examined in the absence and presence of IL-1 $\beta$. In addition, the expression of VE-cadherin junction protein was examined.

\section{Materials and methods}

Cell culture. The HRGECs and HUVECs were obtained and cultured, as previously described $(21,22)$. The HRGECs and HUVECs between passages five and seven were plated onto 6 -well plates and grown to confluence prior to exposure to IL-1 $\beta$ (Invitrogen Life Technologies, Carlsbad, CA, USA).

Reverse transcription quantitative polymerase chain reaction (RT-qPCR). The confluent HRGECs and HUVECs, seeded onto 6 -well plates $\left(2.5 \times 10^{5} /\right.$ well $)$, were treated with $10 \mathrm{ng} / \mathrm{ml}$ IL-1 $\beta$ and collected after $0,1,6,12$ and $24 \mathrm{~h}$. Total RNA ( $25 \mu \mathrm{l}$; four samples from each treatment group) was extracted using an E.Z.N.A Total RNA kit II (Omega Bio-tek, Inc., Norcross, GA, USA) according to the manufacturer's instructions. RT was performed using a RevertAid First Strand cDNA synthesis kit (Thermo Fisher Scientific, Waltham, MA, USA) and qPCR was performed using SYBR Premix Ex TaqII (Takara Bio, Inc., Otsu, Japan). The sequences of the primers used for RT-qPCR are summarized in Table I. The reactions were performed at $50^{\circ} \mathrm{C}$ for $2 \mathrm{~min}$ and $95^{\circ} \mathrm{C}$ for $2 \mathrm{~min}$, followed by 40 cycles of $95^{\circ} \mathrm{C}$ for $15 \mathrm{sec}$ and $60^{\circ} \mathrm{C}$ for $1 \mathrm{~min}$. The gene expression levels of the samples were assessed by comparing the relative expression level of each gene with the internal reference, GAPDH (Abcam, Cambridge, UK).

Western blotting. The HRGECs and HUVECs were lysed using radioimmunoprecipitation assay buffer (Santa Cruz Biotechnology, Inc., Dallas, TX, USA) supplemented with the protease inhibitors aprotonin $(1 \mu \mathrm{g} / \mathrm{ml}$; Santa Cruz Biotechnology Inc.), leupeptin $(10 \mu \mathrm{g} / \mathrm{ml}$; Sigma-Aldrich,
St. Louis, MO, USA) and dimethyl sulfoxide (1 mM; Santa Cruz Biotechnology, Inc.). Equal quantities of the protein were electrophoresed through a 10\% SDS-PAGE gel (Beyotime Institute of Biotechnology, Haimen, China) and were then transferred onto a nitrocellulose membrane (Sigma-Aldrich). The membrane was blocked in Tris-buffered saline and Tween 20 (TBST; Cell Signaling Technology, Inc., Danvers, MA, USA) with $2.5 \%$ non-fat milk proteins at $37^{\circ} \mathrm{C}$ for $1.5 \mathrm{~h}$ prior to incubation with primary antibodies overnight at $4^{\circ} \mathrm{C}$. Following washing three times with TBST, the membrane was incubated with secondary antibody at $37^{\circ} \mathrm{C}$ for $2 \mathrm{~h}$. The primary antibodies used were rabbit anti-human VE-cadherin polyclonal antibody (Abcam; 1:1,000) and rabbit anti-human GAPDH polyclonal antibody (Abcam; 1:2,000). The secondary antibody used was HRP-linked goat anti-rabbit IgG antibody (Cell Signaling Technology Inc.; 1:2,000). The blots were developed using enhanced chemiluminescence reagents (Millipore, Billerica, MA, USA) and the relative intensities of the immunoblots were quantified using ImageJ software (National Institutes of Health, Bethesda, MA, USA).

Fluorescein isothiocyanate (FITC)-dextran Transwell assay. The HUVEC and HRGEC monolayers were plated ( $2 \times 10^{4} /$ well) onto the insert of the Transwell (Corning, Inc., Kennebunk, ME, USA) and cultured until confluent. The chambers were then washed with Hepes medium (Beyotime Institute of Biotechnology) and FITC-dextran (Invitrogen Life Technologies) was added to the top chamber. After $4 \mathrm{~h}$, the samples were removed from the bottom chamber and read in a fluorometer (Gemini EM; Molecular Devices, Sunnyvale, CA, USA) at an excitation of $485 \mathrm{~nm}$ and an emission of $520 \mathrm{~nm}$. The data presented as the mean of three independent experiments.

Electric cell-substrate impedance sensing (ECIS) analysis. The endothelial cell intercellular resistance across cell monolayers was measured using an ECIS technique (ECIS model 1600; Applied BioPhysics, Troy, NY, USA). Briefly, 8-well ECIS arrays (8W10E+) were coated with fibronectin (Invitrogen Life Technologies). The HRGECs and HUVECs were plated at a confluent density to form monolayers directly on top of the electrodes. Following treatment with IL-1 $\beta$, an alternating current was applied across the electrodes and the electrical resistance was recorded. Data plots were constructed, representative of triplicate experiments, with each graph representing resistance readings from a separate well at 40 distinct electrodes per well.

Immunofluorescence. The HUVEC and HRGEC monolayers, grown on fibronectin-coated glass chamber slides (Electron Microscopy Sciences, Hatfield, PA, USA), were exposed to $10 \mathrm{ng} /$ $\mathrm{ml}$ IL-1 $\beta$. After $12 \mathrm{~h}$, the media was aspirated and the monolayers were washed with PBS containing $100 \mathrm{mM}$ L-glycine, fixed with $4 \%$ paraformaldehyde and washed with in PBS for 10 min three times. Immunofluorescence was performed by staining the monolayers with a primary antibody against human VE-cadherin (Abcam) at a dilution of 1:500 overnight at $4^{\circ} \mathrm{C}$ and a rhodamine-labeled secondary antibody (1:200) for $30 \mathrm{~min}$. Images of the slides were captured using an Olympus LCX100 imaging system (Olympus, Tokyo, Japan) with an excitation wavelength of $546 \mathrm{~nm}$. 
Table I. Primer sequences.

\begin{tabular}{|c|c|c|c|}
\hline Gene & Sequence $\left(5^{\prime}-3^{\prime}\right)$ & Size (bp) & Temperature $\left({ }^{\circ} \mathrm{C}\right)$ \\
\hline \multicolumn{4}{|l|}{ GAPDH } \\
\hline Sense & TGATGACATCAAGAAGGTGGTGAAG & \multirow{2}{*}{240} & \multirow{2}{*}{$57.9-63.1$} \\
\hline Antisense & TCCTTGGAGGCCATGTGGGCCAT & & \\
\hline \multicolumn{4}{|l|}{ VE-cadherin } \\
\hline Sense & GCGACTACCAGGACGCTTTCA & \multirow{2}{*}{150} & \multirow{2}{*}{59.5} \\
\hline Antisense & CATGTATCGGAGGTCGATGGTG & & \\
\hline
\end{tabular}

bp, base pairs; VE, vascular endothelial.

\section{A}
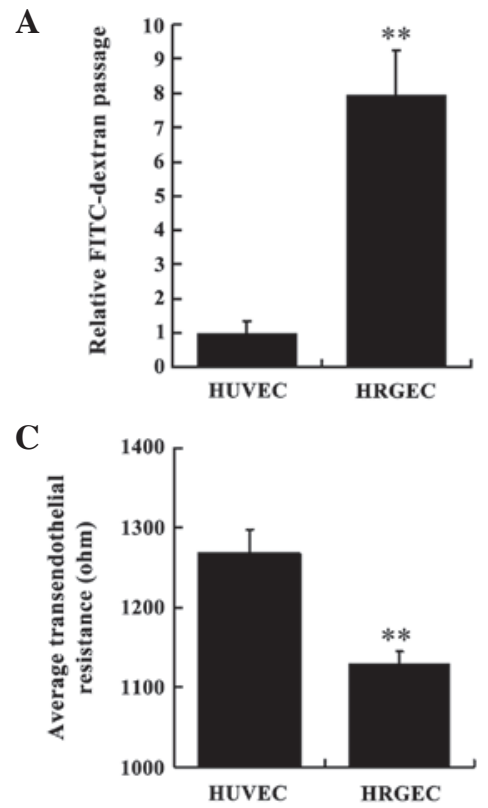

B

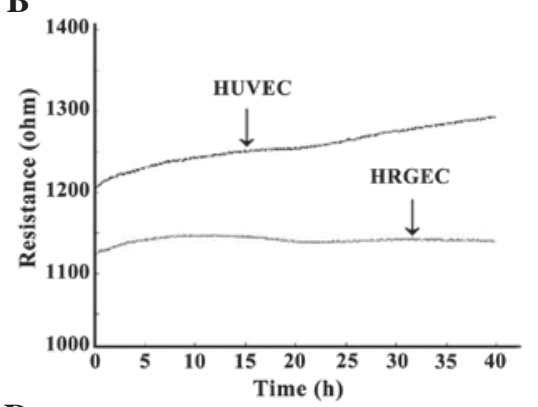

D

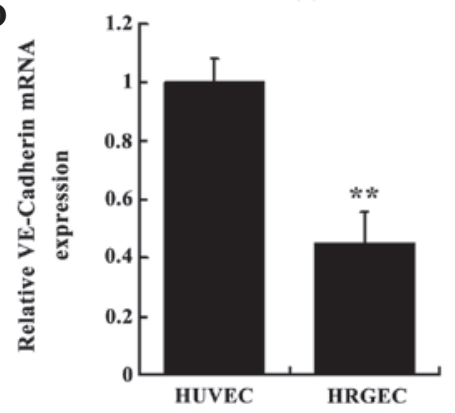

Figure 1. Comparison of the vascular permeability of HUVECs and HRGECs. (A) Transwell migration assay of the HUVEC and HRGEC monolayers $(\mathrm{n}=6),{ }^{* *} \mathrm{P}<0.01$, compared with the HUVEC. (B) Real-time TEER measurement of the HUVEC and HRGEC monolayers using an electric cell-substrate impedance sensing system. (C) Bar graph of the average TEER ( $n=4),{ }^{* *} \mathrm{P}<0.01$, compared with the HUVEC. (D) Relative mRNA expression of VE-cadherin in the HUVECs and HRGECs by reverse transcription polymerase chain reaction $(\mathrm{n}=4),{ }^{* *} \mathrm{P}<0.01$, compared with the HUVEC. Values are expressed as the mean \pm standard error of the mean. HUVEC, human umbilical vein endothelial cell; HRGEC, human glomerular endothelial cell; VE, vascular endothelium; FITC, fluorescein isothiocyanate; TEER, transendothelial electrical resistance.

Statistical analysis. Statistical significances were assessed using a paired-samples t-test. Statistical analysis was performed using the SPSS 17.0 statistical software package (SPSS Inc., Chicago, IL, USA). $\mathrm{P}<0.05$ was considered to indicate a statistically significant difference.

\section{Results}

HRGECs demonstrate higher permeability compared with HUVECs. The permeability of the HUVECs and HRGECs cultured on Transwell filters was assayed using a FITC-Dextran tracer, ranging between 4 and $150 \mathrm{kDa}$ in molecular weight. The FITC-Dextran, which passed through the monolayer of the HRGECs was 7.6-fold higher compared with that of the HUVECs (Fig. 1A), indicating that the fenestrated HRGECs were more permeable than the non-fenestrated HUVECs. To identify subtle alterations of vascular permeability, an ECIS system was used to enable real-time measurements of the resistance of a monolayer of endothelial cells (23). In an ECIS circuit, the current flows across the cell monolayer and the gaps between the cells function as resistors (24). The ECIS analysis indicated that the HRGEC monolayers maintained a transendothelial electrical resistance (TEER) of $1128.3 \pm 16.97 \mathrm{ohm}$ up to $40 \mathrm{~h}$, which was significantly lower compared with the resistance of $1267.6 \pm 29.78 \mathrm{ohm}$ observed in the HUVEC monolayers $(\mathrm{P}<0.01$; Fig. 1B and $\mathrm{C})$, which may be due to the fenestration and weak gap junctions of the HRGECs. VE-cadherin is the endothelial cell-specific component of adherens junctions and is essential for the maintenance of endothelial cell barrier integrity (25). The present study examined the mRNA level of VE-cadherin by RT-qPCR and found that its expression in the HRGECs was significantly lower compared with that in the HUVECs $(\mathrm{P}<0.01$; Fig. 1D). 
A

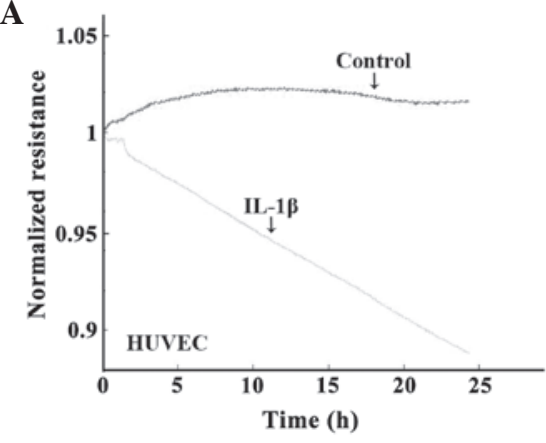

C

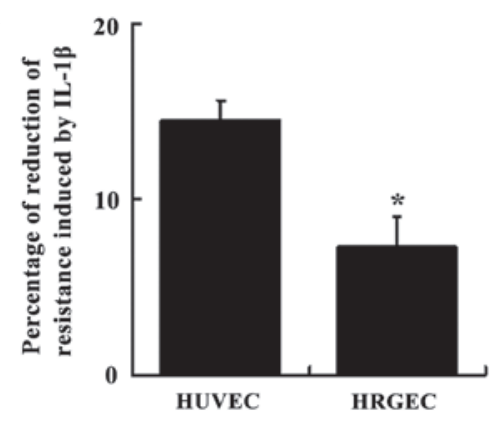

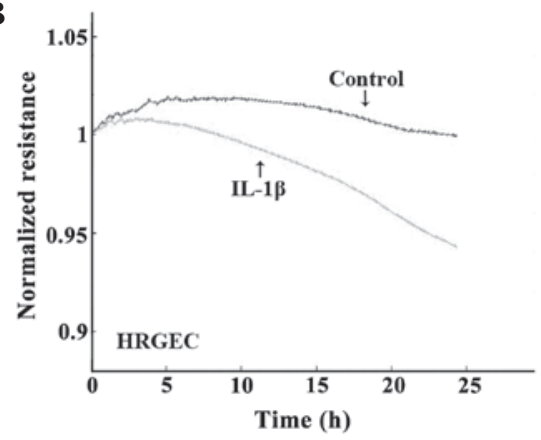

D

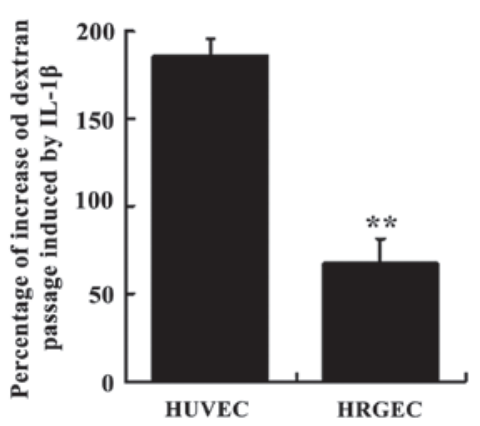

Figure 2. Effects of IL1- $\beta$ on the permeability of HUVECs and HRGECs. (A) Representative image of TEER measurement of the HUVECs treated with IL1- $\beta$. (B) Representative plots of the TEER of the HRGECs treated with IL1- $\beta$. (C) Average percentage reduction of TEER in the HUVEC and HRGEC monolayers induced by IL1- $\beta(n=3),{ }^{*} \mathrm{P}<0.05$, compared with the HUVEC. (D) Average percentage increase in FITC-Dextran passage through the HUVEC and HRGEC monolayers in a Transwell permeability assay $(n=6),{ }^{* *} \mathrm{P}<0.01$, compared with the HUVEC. Values are expressed as the mean \pm standard error of the mean. HUVEC, human umbilical vein endothelial cell; HRGEC, human glomerular endothelial cell; IL, interleukin; FITC, fluorescein isothiocyanate; TEER, transendothelial electrical resistance.

HRGECs are less responsive to $I L-1 \beta$ treatment compared with HUVECs. Vascular inflammation and increased endothelial permeability are critical factors causing kidney disease and renal failure (26). Cytokines, including TNF- $\alpha$ and IL-1 $\beta$ can disrupt junctional molecules and increase endothelial permeability (19). Using the ECIS system, the present study examined the effects of IL-1 $\beta$ on the TEER of the HUVEC and HRGEC monolayers. At a concentration of $10 \mathrm{ng} / \mathrm{ml}$, IL-1 $\beta$ induced a significant decrease in TEER in the two cell types (Fig. 2A and B). The reduction of TEER in the HRGECs $(7.89 \%)$ was significantly lower compared with that in the HUVECs $(14.26 \%$; $\mathrm{P}<0.01$; Fig. $2 \mathrm{C}$ ), suggesting that the permeability of the HRGEC was less responsive to IL-1 $\beta$. A Transwell permeability assay was also performed on the cell monolayers following IL-1 $\beta$ treatment. As shown in Fig. 2D, the FITC-Dextran passage was increased by $186 \%$ in the HUVEC monolayers, however it was increased by only $65 \%$ in the HRGEC monolayers $(\mathrm{P}<0.01)$. Therefore, following treatment with the same concentration of IL-1 $\beta$, the increase of permeability in the HRGEC monolayers was significantly lower.

VE-cadherin expression is increased in HRGECs and decreased in HUVECs following IL-1 $\beta$ treatment. The function of the endothelial barrier requires the adhesive activity of VE-cadherin (27). The mRNA levels of VE-cadherin in the HUVECs and HRGECs treated with IL-1 $\beta$ were examined at different time points by RT-qPCR. The expression of VE-cadherin was downregulated in the HUVECs following incubation with IL-1 $\beta$ for $12 \mathrm{~h}(\mathrm{P}<0.05$; Fig. 3A). However, the expression of VE-cadherin was increased in the HRGECs
$(\mathrm{P}<0.01$; Fig. 3C). Subsequently, the present study examined the protein expression of VE-cadherin by western blot analysis. The protein level of VE-cadherin was decreased in the HUVECs (Fig. 3B), but increased in the HRGECs following IL-1 $\beta$ treatment (Fig. 3D). In addition, the distribution of VE-cadherin in the HUVECs and HRGECs were examined by immunofluorescence. VE-cadherin was detected on the membrane in the HUVECs and HRGECs (Fig. 4A and B). Following IL-1 $\beta$ treatment, VE-cadherin staining was decreased in the HUVECs (Fig. 4C) and increased in the HRGECs (Fig. 4D). Therefore, VE-cadherin was differentially regulated in the HUVECs and HRGECs in response to IL-1 $\beta$.

\section{Discussion}

Renal GECs are a unique microvascular cell type, which are morphologically and functionally specialized to support the selective filtration of plasma (3). However, the biological properties of GECs remain to be fully elucidated. In the present study, HRGECs demonstrated higher permeability, lower TEER and lower expression levels of VE-cadherin compared with HUVECs. IL- $1 \beta$ increased the permeability and decreased the TEER of the HRGECs, but to a lesser extent compared with the HUVECs. Following IL-1 $\beta$ treatment, the expression of VE-cadherin was increased in the HRGECs and decreased in the HUVECs. The data revealed the distinctive characteristics of HRGECs.

GECs differ from other types of endothelial cell as they are particularly flattened and fenestrated (3). These structures cause the glomerular capillary wall to be highly permeable to water and solutes (3). In addition to the fenestrated pores in HRGECs, 

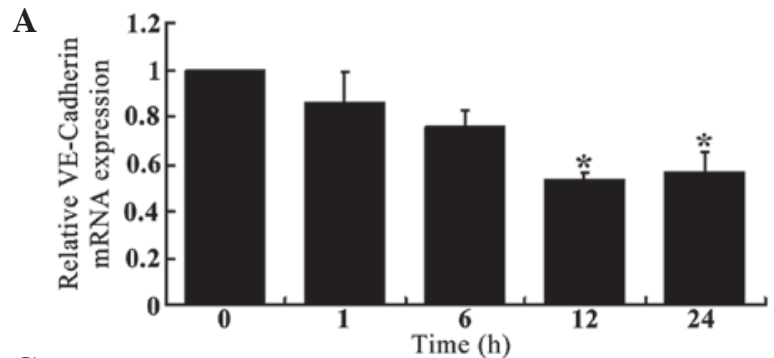

C

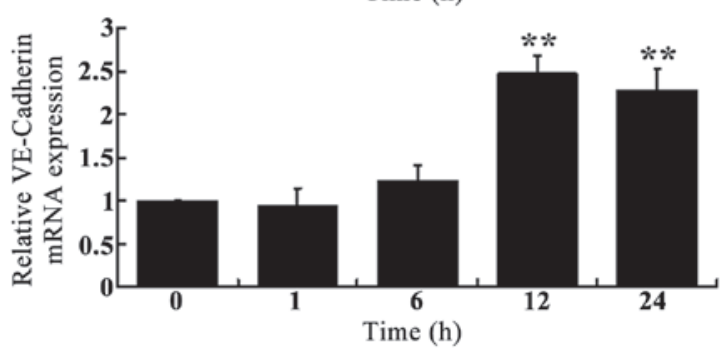

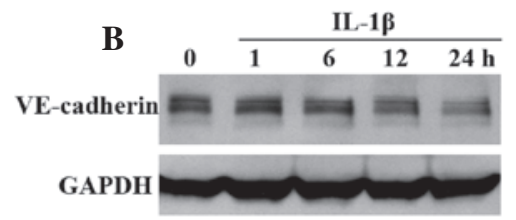

D

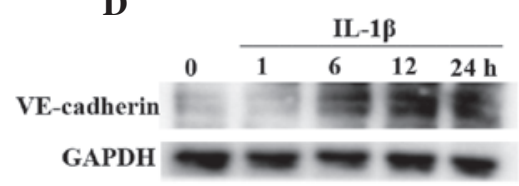

Figure 3. Expression of VE-cadherin in the HUVECs and HRGECs in response to IL1- $\beta$. (A) Time course of the mRNA expression of VE-cadherin in the HUVECs in response to IL1- $\beta(n=4),{ }^{*} \mathrm{P}<0.05$ vs. non-treatment group. (B) Representative immunoblots of VE-cadherin and GAPDH from protein samples of HUVECs treated with IL1- $\beta$ at different time points. (C) Time course of the mRNA expression of VE-cadherin in HRGECs in response to IL1- $\beta$ ( $n=4$ ), ${ }^{* *} \mathrm{P}<0.01$ vs. non-treatment group. (D) Representative immunoblots of VE-cadherin and GAPDH from protein samples of HRGECs treated with IL1- $\beta$ at different time points. Values are expressed as the mean \pm standard error of the mean. HUVEC, human umbilical vein endothelial cell; HRGEC, human glomerular endothelial cell; IL, interleukin; VE, vascular endothelium.
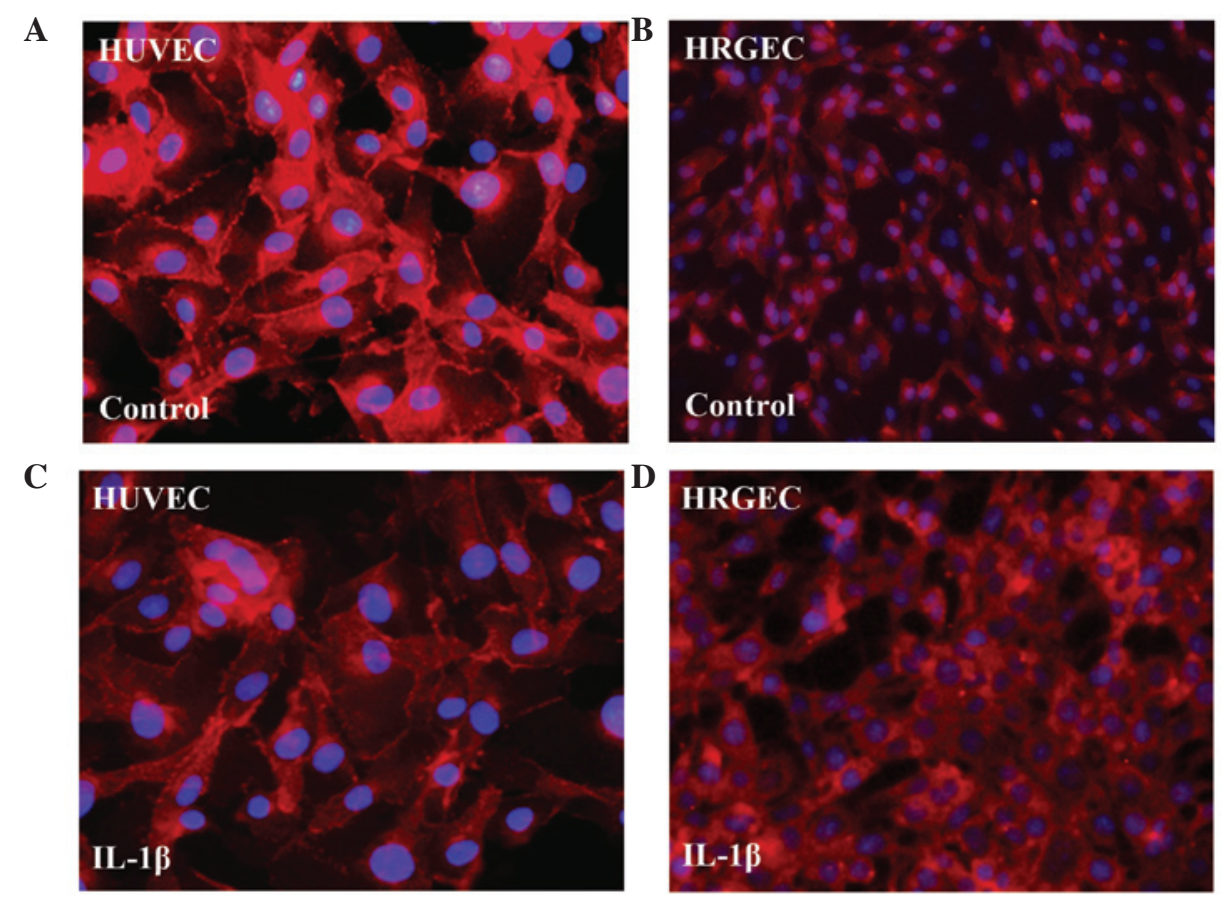

Figure 4. Effects of IL1- $\beta$ on the distribution of VE-cadherin in HUVECs and HRGECs. Immunofluoresence staining of VE-cadherin in (A) HUVECs, (B) HRGECs, (C) HUVECs treated with IL1- $\beta$ and (D) HRGECs treated with IL1- $\beta$ (magnification, x100). HUVEC, human umbilical vein endothelial cell; HRGEC, human glomerular endothelial cell; IL, interleukin; VE, vascular endothelium.

the endothelial cell-cell junctions control paracellular permeability, particularly for the passage of larger molecules $(28,29)$. In the present study, the higher permeability and lower TEER of the HRGECs may be due to fenestration and junction integrity. VE-cadherin is a transmembrane adhesion molecule, which bridges adjacent endothelial cells (30). It is associated with $\beta$ - and $\gamma$-catenin, which bind $\alpha$-catenin, an actin-binding protein (30). This VE-cadherin-catenin complex is essential for the maintenance of endothelial cell barrier integrity (27). The present study demonstrated that the expression of VE-cadherin was lower in the HRGECs compared with the HUVECs, implicating relatively fewer adherens junctions between the HRGECs.

In addition to ultrafiltration, HRGECs form the interface between the blood and the underlying tissues, interact with blood flow and regulate vasomotor tone, as with other endothelial cells (6). Circulating pro-inflammatory cytokines directly stimulate the endothelium and disrupt endothelial cell-cell junctions (7). TNF- $\alpha$, IL- $1 \beta$ and IFN- $\gamma$ increase 
the permeability of HUVEC monolayers in a dose- and time-dependent manner (31) and IL-1 $\beta$ induces barrier dysfunction in endothelial cells from different vascular beds (32-34). In the present study, the HRGECs and HUVECs exhibited an increase in permeability and a decrease in TEER in response to IL-1 $\beta$. However, the barrier function of the HRGECs was affected less compared with that of the HUVECs, suggesting a cell-subytpe specific mechanism may be involved in regulating inflammatory responses in HRGECs. To the best of our knowledge, the present study is the first to compared the effects of IL-1 $\beta$ on HRGECs with HUVECs.

VE-cadherin-mediated adhesion is important for the control of vascular permeability and leukocyte extravasation (30). The data obtained in the present study revealed that the expression of VE-cadherin was downregulated in the HUVECs, but upregulated in the HRGECs following treatment with IL-1 $\beta$. The increased expression of VE-cadherin may improve barrier integrity and partially compensate the permeability of the HRGECs induced by IL-1 $\beta$. All three types of inter-endothelial junctions, including tight junctions, adherens junctions and gap junctions, have been identified in GECs $(28,29)$. In addition to the effects on VE-cadherin and adherens junctions, IL-1 $\beta$ disrupts tight junctions and gap junctions by regulating claudin-1 and connexin $43(35,36)$. Therefore, IL-1 $\beta$ may induce the permeability of HRGECs through VE-cadherin-independent pathways.

Endothelial cells from different vascular beds balance local hemostasis and exhibit marked heterogeneity in structure and function $(37,38)$. Significant differences in the levels of basal and inducible gene expression have been detected in cultured endothelial cells isolated from specific organs (39). In response to oxidized low density lipoproteins, TNF- $\alpha$ and IL- $1 \beta$, human coronary artery endothelial cells and human saphenous vein endothelial cells exhibit distinct gene expression profiles (40). In the present study, the expression of VE-cadherin was differentially regulated by IL- $1 \beta$ in the HRGECs and HUVECs, which is in accordance with the concept of endothelial cell heterogeneity. Each subtype of endothelial cells may have distinct biological properties, which differ from other endothelial cells (41). The detailed characteristics of HRGECs require further investigation to understand their roles in health and disease.

In conclusion, the present study characterized the permeability of HRGECs and analyzed their responses to IL-1 $\beta$, a pro-inflammatory cytokine. These findings revealed the distinct features of GECs and, therefore, assist in understanding the pathophysiological mechanisms underlying damage to the glomerular filtration barrier.

\section{Acknowledgements}

This study was supported by grants from the Science and Technology Development Plan of Shandong Province (no. 2013GSF11805) and the Shandong Taishan Scholarship (Ju Liu). The authors would like to thank Dr Meiyu Cui, Dr Jing Sun and Dr Jia Wang for their technical support.

\section{References}

1. Miner JH: Glomerular basement membrane composition and the filtration barrier. Pediatr Nephrol 26: 1413-1417, 2011.
2. Levidiotis V and Power DA: New insights into the molecular biology of the glomerular filtration barrier and associated disease. Nephrology (Carlton) 10: 157-166, 2005.

3. Obeidat $\mathrm{M}$ and Ballermann BJ: Glomerular endothelium: a porous sieve and formidable barrier. Exp Cell Res 318: 964-972, 2012.

4. Haraldsson BS: The endothelium as part of the integrative glomerular barrier complex. Kidney Int 85: 8-11, 2014.

5. Haraldsson B, Nystrom J and Deen WM: Properties of the glomerular barrier and mechanisms of proteinuria. Physiol Rev 88: 451-487, 2008.

6. Savage CO: The biology of the glomerulus: endothelial cells. Kidney Int 45: 314-319, 1994.

7. Stylianou E and Saklatvala J: Interleukin-1. Int J Biochem Cell Biol 30: 1075-1079, 1998.

8. Al-Sadi R, Boivin M and Ma T: Mechanism of cytokine modulation of epithelial tight junction barrier. Front Biosci (Landmark Ed) 14: 2765-2778, 2009

9. Yamanaka N and Shimizu A: Role of glomerular endothelial damage in progressive renal disease. Kidney Blood Press Res 22: 13-20, 1999.

10. Lee LK, Meyer TW, Pollock AS and Lovett DH: Endothelial cell injury initiates glomerular sclerosis in the rat remnant kidney. J Clin Invest 96: 953-964, 1995.

11. van de Veerdonk FL and Netea MG: New Insights in the Immunobiology of IL-1 Family Members. Front Immunol 4: 167, 2013.

12. Zhao R, Zhou H and Su SB: A critical role for interleukin-1beta in the progression of autoimmune diseases. Int Immunopharmacol 17: 658-669, 2013.

13. Himmelfarb J, Le P, Klenzak J, Freedman S, McMenamin ME and Ikizler TA: Impaired monocyte cytokine production in critically ill patients with acute renal failure. Kidney Int 66: 2354-2360, 2004.

14. Boraschi D, Bossu P, Macchia G, Ruggiero P and Tagliabue A: Structure-function relationship in the IL-1 family. Front Biosci 1: d270-d308, 1996.

15. Auron PE: The interleukin 1 receptor: ligand interactions and signal transduction. Cytokine Growth Factor Rev 9: 221-237, 1998.

16. Dinarello CA: The IL-1 family and inflammatory diseases. Clin Exp Rheumatol 20: S1-S13, 2002.

17. Voronov E, Carmi Y and Apte RN: Role of IL-1-mediated inflammation in tumor angiogenesis. Adv Exp Med Biol 601: 265-270, 2007.

18. Wong D, Dorovini-Zis K and Vincent SR: Cytokines, nitric oxide and cGMP modulate the permeability of an in vitro model of the human blood-brain barrier. Exp Neurol 190: 446-455, 2004

19. Nooteboom A, Van Der Linden CJ and Hendriks T: Tumor necrosis factor-alpha and interleukin-lbeta mediate endothelial permeability induced by lipopolysaccharide-stimulated whole blood. Crit Care Med 30: 2063-2068, 2002.

20. Ganter MT, Roux J, Miyazawa B, et al: Interleukin-1beta causes acute lung injury via alphavbeta5 and alphavbeta6 integrin-dependent mechanisms. Circ Res 102: 804-812, 2008.

21. Du P, Fan B, Han H, et al: NOD2 promotes renal injury by exacerbating inflammation and podocyte insulin resistance in diabetic nephropathy. Kidney Int 84: 265-276, 2013.

22. Liu J, Yuan L, Molema G, et al: Vascular bed-specific regulation of the von Willebrand factor promoter in the heart and skeletal muscle. Blood 117: 342-351, 2011.

23. Becker PM, Verin AD, Booth MA, Liu F, Birukova A and Garcia JG: Differential regulation of diverse physiological responses to VEGF in pulmonary endothelial cells. Am J Physiol 281: L1500-L1511, 2001.

24. Birukova AA, Birukov KG, Smurova K, et al: Novel role of microtubules in thrombin-induced endothelial barrier dysfunction. FASEB J 18: 1879-1890, 2004.

25. Hordijk PL, Anthony E, Mul FP, Rientsma R, Oomen LC and Roos D: Vascular-endothelial-cadherin modulates endothelial monolayer permeability. J Cell Sci 112 (Pt 12): 1915-1923, 1999.

26. Ponnuchamy B and Khalil RA: Cellular mediators of renal vascular dysfunction in hypertension. Am J Physiol Regul Integr Comp Physiol 296: R1001-R1018, 2009.

27. Dejana E and Giampietro C: Vascular endothelial-cadherin and vascular stability. Curr Opinion Hematol 19: 218-223, 2012.

28. Deen WM: What determines glomerular capillary permeability? J Clin Invest 114: 1412-1414, 2004.

29. Obeidat M, Obeidat M and Ballermann BJ: Glomerular endothelium: a porous sieve and formidable barrier. Exp Cell Res 318: 964-972, 2012. 
30. Vestweber D: VE-cadherin: the major endothelial adhesion molecule controlling cellular junctions and blood vessel formation. Arterioscler Thromb Vasc Biol 28: 223-232, 2008.

31. Burke-Gaffney A and Keenan AK: Modulation of human endothelial cell permeability by combinations of the cytokines interleukin-1 alpha/beta, tumor necrosis factor-alpha and interferon-gamma. Immunopharmacology 25: 1-9, 1993.

32. Cromer WE, Zawieja SD, Tharakan B, Childs EW, Newell MK and Zawieja DC: The effects of inflammatory cytokines on lymphatic endothelial barrier function. Angiogenesi 2013.

33. Qin W,Lu W,Li H, et al: Melatonin inhibits ILlbeta-induced MMP9 expression and activity in human umbilical vein endothelial cells by suppressing NF-kappaB activation. J Endocrinol 214: 145-153, 2012.

34. Rigor RR, Beard RS, Jr, Litovka OP and Yuan SY: Interleukin-1beta-induced barrier dysfunction is signaled through PKC-theta in human brain microvascular endothelium. Am J Physiol. Cell Physiol 302: C1513-C1522, 2012.

35. Duffy HS, John GR, Lee SC, Brosnan CF and Spray DC: Reciprocal regulation of the junctional proteins claudin-1 and connexin 43 by interleukin-1beta in primary human fetal astrocytes. J Neurosci 20: RC114, 2000.
36. Qi J, Chi L, Bynum D and Banes AJ: Gap junctions in IL-1beta-mediated cell survival response to strain. J Appl Physiol 110: 1425-1431, 2011.

37. Kinjo T, Takashi M, Miyake K and Nagura H: Phenotypic heterogeneity of vascular endothelial cells in the human kidney. Cell Tissue Res 256: 27-34, 1989.

38. Atkins GB, Jain MK and Hamik A: Endothelial differentiation: molecular mechanisms of specification and heterogeneity. Arterioscler Thromb Vasc Biol 31: 1476-1484, 2011.

39. Aird WC: Mechanisms of endothelial cell heterogeneity in health and disease. Circ Res 98: 159-162, 2006.

40. Deng DX, Tsalenko A, Vailaya A, et al: Differences in vascular bed disease susceptibility reflect differences in gene expression response to atherogenic stimuli. Cir Res 98: 200-208, 2006.

41. Molema G and Aird WC: Vascular heterogeneity in the kidney. Semin Nephrol 32: 145-155, 2012. 